\title{
Plant Disease Classification using Deep Learning Google Net Model
}

\author{
Satwinder Kaur, Garima Joshi, Renu Vig
}

\begin{abstract}
Plant diseases have been a major crisis that is disturbing the food production. So there is a need to provide proper procedures for plant disease detection at its growing age and also during harvesting stage. Timely disease detection can help the user to respond instantly and sketch for some defensive actions. This detection can be carried out without human intervention by using plant leaf images. Deep learning is progressively best for image detection and classification. In this effort, a deep learning based GoogleNet architecture is used for plant diseases detection. The model is trained using public database of 54,306 images of 14 crop varieties and their respective diseases. It achieves $97.82 \%$ accuracy for 14 crop types making it capable of further deployment in a crop detection and protection application.
\end{abstract}

Index Terms: Deep learning, GoogleNet, Plant disease detection

\section{INTRODUCTION}

Safety measure in food production is a major concern because the total population in world is expected to grow over more than 9.7 billion by 2050. There are many cultivation losses due to pathogens such as microbes, virus and fungus. So to minimize these losses induced in crops during growth and harvest, advanced disease detection and prevention techniques for crops are exigent. Diagnoses of plant disease by human specialist require high degree of proficiency because they must have experience in detecting large variety of disease symptoms [1]. Even experienced plant pathologists may fail to accurately detect specific diseases and diagnose them. Therefore, with progress in digital cameras and information technology, expert systems in farming have been used for crop management. Disease diagnostics and treatment shall help in improving production capacity of plants [2]. Hence, a strategy that automates the classification of diseases using images is necessary. The advantage of computerized system for the analysis of plant diseases is that it helps the agronomist to perform such diagnosis through optical observation [3]. The chief goal of these approaches is to automatically identify the diseases in order to provide suitable treatment in time [4].

In recent times, tools based on cell phones have grown rapidly, smart phones offer efficient portable solution to identify diseases due to their computing power and high degree displays.

Revised Manuscript Received on July 02, 2019.

Satwinder Kaur, Garima Joshi, Renu Vig Panjab University, Chandigarh, India

The combined capabilities of mobile devices along with appropriate algorithm can lead to automated disease diagnoses. Moreover, these approaches are based on machine learning and computer vision to classify diseases using only images of plants [5].

\section{RELATED WORKS}

Various methods are deployed for plant disease identification applying computer vision. Koushik et al. reviewed the claim for developing a fast, cost efficient, and consistent system for farming. They described presently the technologies that include spectroscopic and image capturing based plant disease detection methods for monitoring strength and disease identification in plants under field conditions [7]. In [8-10], image processing disease recognition approach was used for plant disease diagnostics. Chaudhary et al. extracted color features, disease spots were identified by extracting some attributes such as shape feature method. Patil and Bodhe used segmentation to resolve leaf area and triangle thresholding for affected area for disease identification in sugarcane leaves getting the average accuracy of $98.60 \%$ [12]. Furthermore, extracting texture feature is also used in plant disease detection. Patil and Kumar projected model for plant disease detection using all three color, shape and texture features. This collective color extraction is used on detecting diseases of Soyabean leaves. Arrangement of all these features leads to a robust system for plant image classification [13]. Recently in 2018, a large, deep convolution neural network (CNN) was developed for plant disease detection using (LVQ) algorithm [14]. Too et al. detected 26 diseases with a fully connected CNN approach. The presentation of model was $99.75 \%$. However, the capability to estimate the exact plant disease pair was limited [15].

With the help of literature review it can be concluded that to improve the accuracy, researchers have studied many methods for plant diseases diagnoses based on conventional machine learning such as k-nearest neighbor, and support vector machine (SVM). In the following years, deep CNN decreased the error rate and enhanced the accuracy. While training of neural networks take much time but the trained models can classify images very simply and rapidly. CNN contains one of the most powerful techniques for performance of pattern detection in an application with huge quantity of data. In this paper, GoogleNet based CNN architecture is trained and assessed to perform an automatic plant disease classification system. 


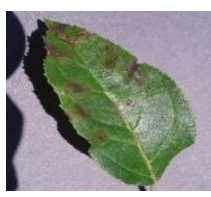

1.Apple Scab

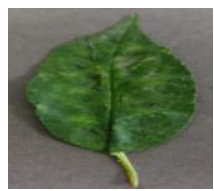

7.Cherrypowdery

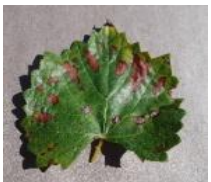

13.Grape spot

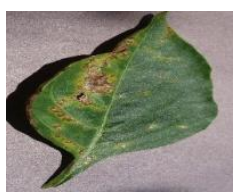

19.Pepperbacterial spot

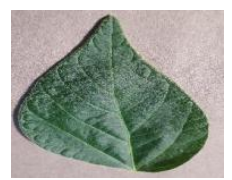

25.Soyabeanhealthy

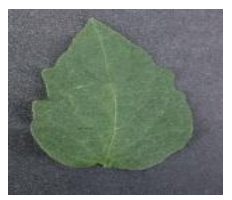

31.Tomato healthy

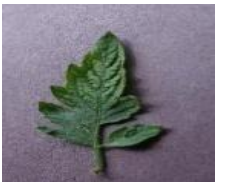

37.Tomato virus

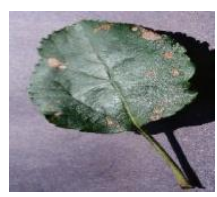

2.Apple black rot

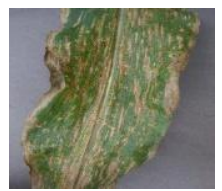

8.Corn leaf spot

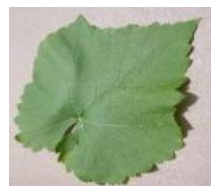

14.Grape healthy

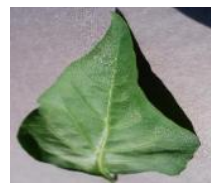

20.Pepper healthy

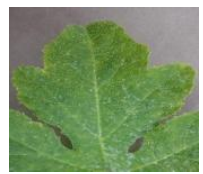

26.Squashpowder mildew

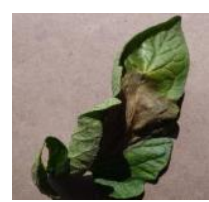

32.Tomato late blight

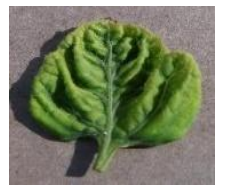

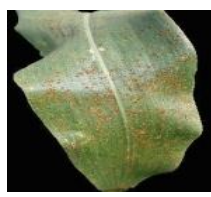

9.Corn rust
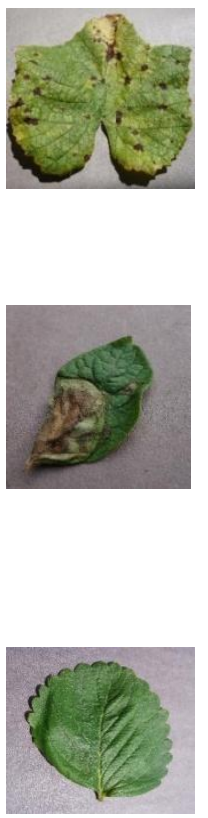
27.Strawberry healthy include information regarding combining the extracted features into probability and class labels. The last fully connected is of same size as the number of classes.
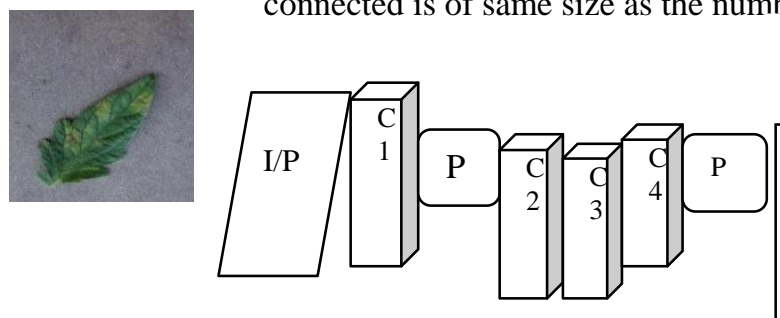

$\mathrm{IM}$

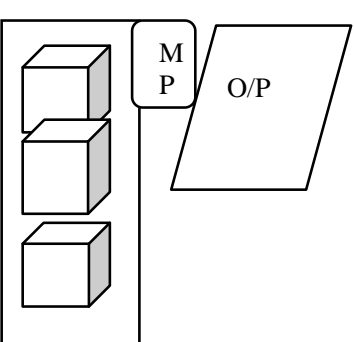

Fig 2: GoogleNet architecture (I/P-input, IM-inception module, C-convolutional, P-polling, MP-max pooling, O/P-output)

38.Tomato yellow virus

In order to ensure faster learning, the learning rate of the fully connected layer is increased. Also the learning rates in earlier layers if set is zero can congeal the weights of earlier layers in the network. Thus during re-training, it does not modify the parameters of the frozen layers. This significantly speeds up network training process. In case of smaller dataset, freezing previous network

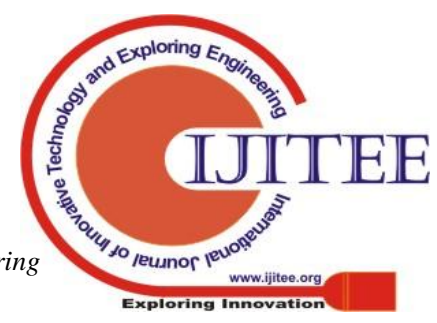


layers also avoids over fitting the new dataset.

Table 1: GoogleNet Training Validation Parameters

\begin{tabular}{|r|l|l|}
\hline S. No. & Prameter & Value \\
\hline 1. & Input Layer size & $\mathbf{2 2 4 \times 2 2 4 \times 3}$ \\
\hline 2. & Epochs & $\mathbf{6}$ \\
\hline 3. & $\begin{array}{c}\text { Validation } \\
\text { Frequency }\end{array}$ & $\mathbf{3}$ iterations \\
\hline 4. & Learning Rate & $\mathbf{0 . 0 0 0 1}$ \\
\hline 5. & $\begin{array}{c}\text { Image } \\
\text { Augmentation }\end{array}$ & Flipping \\
\hline 6. & Hardware resource & Single GPU \\
\hline
\end{tabular}

The performance of GoogleNet on PlantVillage dataset is analyzed by training the network using transfer learning. Segmentation and feature extraction is not requirement in CNN based deep learning models as they have inbuilt layers to classify the important and unimportant features of a given class of images. The parameters used in training the GoogleNet for PlantVillage dataset is listed in Table 1 are used. The first step is to load the dataset images using augmented image data store. Load the pretrained GoogleNet network and extract the graph from trained network. Replace the final layers and freeze initial layers. Then training the network using training data by augmentation is done and classification accuracy is calculated using validation data.

\section{RESULTS AND DISCUSSIONS}

Table 2 presents the results of 38 diverse classes of plant disease pair. The outcomes are shown in terms of total number of images, accuracy and time. The overall accuracy obtained on PlantVillage dataset improved from $87.32 \%$ (while performing on single CPU by basic CNN architecture) to $97.82 \%$ (in case of GoogleNet performing on a single GPU). In some cases like cherry accuracy achieved is $100 \%$ due to less number of sub-classes as shown in Table 2. A snapshot of the result is shown in Fig 3. All the investigational configurations run for total of 6 epochs. The learning rate starting from 0.01 (in a case of $\mathrm{CNN}$ ) reduced to 0.0001 in GoogleNet. However, the time taken to train the network increased by $40 \%$ in case of GoogleNet.

Table 2: Plant diseases and their classification

\begin{tabular}{|c|c|c|c|c|c|}
\hline $\begin{array}{c}\text { Class } \\
\text { No. }\end{array}$ & $\begin{array}{l}\text { Plant } \\
\text { Name }\end{array}$ & Disease Name & $\begin{array}{l}\text { No. of } \\
\text { images }\end{array}$ & $\begin{array}{l}\text { Time } \\
\text { Taken }\end{array}$ & Accuracy \\
\hline 1 & Apple & Apple scab & 2520 & \multirow{4}{*}{$\begin{array}{c}133 \mathrm{m31} \\
\mathrm{s}\end{array}$} & \multirow{4}{*}{$99.59 \%$} \\
\hline 2 & Apple & Apple black rot & 2484 & & \\
\hline 3 & Apple & Apple cedar rust & 2200 & & \\
\hline 4 & Apple & Apple healthy & 2510 & & \\
\hline 5 & $\begin{array}{c}\text { Blueberr } \\
\mathbf{y}\end{array}$ & Blueberry healthy & 2270 & $23 m 35 s$ & $100 \%$ \\
\hline 6 & Cherry & Cherry healthy & 2282 & \multirow[b]{2}{*}{$95 \mathrm{~m} 21 \mathrm{~s}$} & \multirow[b]{2}{*}{$100 \%$} \\
\hline 7 & Cherry & $\begin{array}{c}\text { Cherry powdery } \\
\text { mildew }\end{array}$ & 2104 & & \\
\hline 8 & Corn & Corn leaf spot & 2052 & \multirow{4}{*}{$188 \mathrm{~m} 3 \mathrm{~s}$} & \multirow{4}{*}{$92.47 \%$} \\
\hline 9 & Corn & Corn common rust & 2317 & & \\
\hline 10 & Corn & Corn healthy & 2324 & & \\
\hline 11 & Corn & Corn leaf blight & 2385 & & \\
\hline 12 & Grape & Grape black rot & 2360 & \multirow[b]{2}{*}{$39 \mathrm{~m} 6 \mathrm{~s}$} & \multirow[b]{2}{*}{$98.74 \%$} \\
\hline 13 & Grape & \begin{tabular}{|cc}
$\begin{array}{c}\text { Grape } \\
\text { measles }\end{array}$ & black \\
\end{tabular} & 2400 & & \\
\hline
\end{tabular}

\section{Training Progress: 18-Apr-2019 10:48:10}

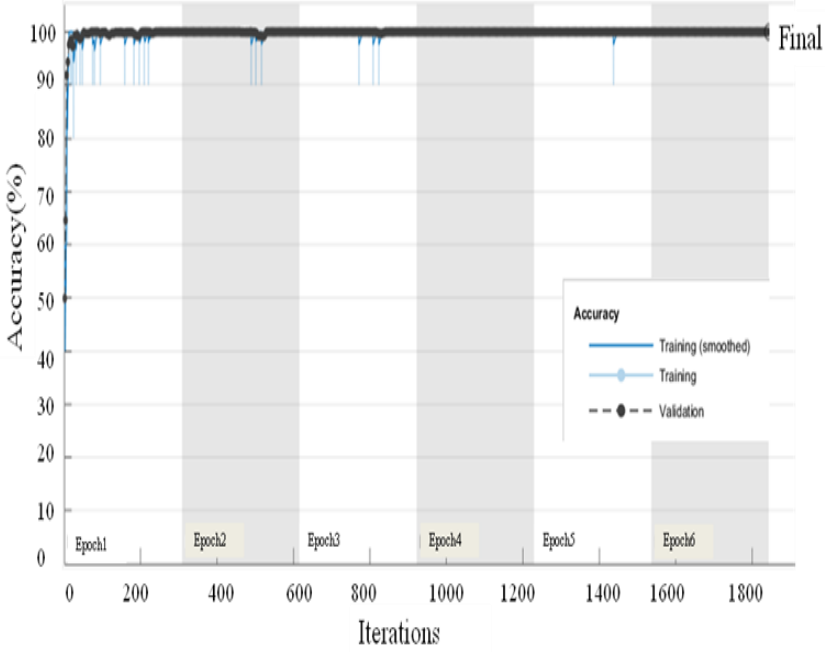

Fig 3: Snapshot of Result after training and validation for Cherry leaf images

\section{CONCLUSION}

In this work, a deep learning model has been trained based on $\mathrm{CNN}$ architectures, for the detection and classification of plant diseases for healthy and diseased plant leaf images. Training/validation of the models is done using 
an openly available dataset. The most successful model architecture is GoogleNet which achieved a success rate of $97.82 \%$. For the experiments performed, GoogleNet has proved to be the best in this case. This can be used as a practical tool for farmers to protect plants against diseases and design a viable additional method that helps to prevent food production loss.

\section{REFERENCES}

[1] Yang, Xin, and Tingwei Guo. "Machine learning in plant disease research." European Journal of Biomedical Research 3, no. 1 6-9.2017.

[2] Mohanty, Sharada P., David P. Hughes, and Marcel Salathé. "Using deep learning for image-based plant disease detection." Frontiers in plant science 7:1419.2017.

[3] Hughes, David, and Marcel Salathé. "An open access repository of images on plant health to enable the development of mobile disease diagnostics." arXiv preprint arXiv: 1511.080602015.

[4] Slodojevic, Srdjan, Marko Arsenovic, Andras Anderla, Dubravko Culibrk, and Darko Stefanovic. "Deep neural networks based recognition of plant diseases by leaf image classification." Computational intelligence and neuroscience 2016.

[5] Rehman, Tanzeel U., Md Sultan Mahmud, Young K. Chang, Jian Jin, and Jaemyung Shin. "Current and future applications of statistical machine learning algorithms for agricultural machine vision systems." Computers and Electronics in Agriculture 156: 585-605. 2019.

[6] Zhang, Shanwen, Haoxiang Wang, Wenzhun Huang, and Zhuhong You. "Plant diseased leaf segmentation and recognition by fusion of superpixel, K-means and PHOG." Optik 157: 866-872.2018.

[7] Nagasubramanian, Koushik, Sarah Jones, Asheesh K. Singh, Arti Singh, Baskar Ganapathysubramanian, and Soumik Sarkar. "Explaining hyperspectral imaging based plant disease identification: 3D CNN and saliency maps." arXiv preprint arXiv: 1804.088312018.

[8] Johannes, Alexander, Artzai Picon, Aitor Alvarez-Gila, Jone Echazarra, Sergio Rodriguez-Vaamonde, Ana Díez Navajas, and Amaia Ortiz-Barredo. "Automatic plant disease diagnosis using mobile capture devices, applied on a wheat use case." Computers and electronics in agriculture 138: 200-209.2017.

[9]Ferentinos, Konstantinos P. "Deep learning models for plant disease detection and diagnosis." Computers and Electronics in Agriculture $145: 311-318.2018$

[10] Iqbal, Zahid, Muhammad Attique Khan, Muhammad Sharif, Jamal Hussain Shah, Muhammad Habib ur Rehman, and Kashif Javed. "An automated detection and classification of citrus plant diseases using image processing techniques: A review." Computers and electronics in agriculture 153: 12-32.2018.

[11] Chaudhary, Piyush, Anand K. Chaudhari, A. N. Cheeran, and Sharda Godara. "Color transform based approach for disease spot detection on plant leaf." International Journal of Computer Science and Telecommunications 3, no. 6: 65-70.2012.

[12]Patil, Sanjay B., and Shrikant K. Bodhe. "Leaf disease severity measurement using image processing." International Journal of Engineering and Technology 3, no. 5: 297-301.2011.

[13] Patil, Jayamala Kumar, and Raj Kumar. "Analysis of content based image retrieval for plant leaf diseases using color, shape and texture features." Engineering in agriculture, environment and food 10, no. 2: 69-78.2017.

[14] Sardogan, Melike, Adem Tuncer, and Yunus Ozen. "Plant Leaf Disease Detection and Classification Based on CNN with LVQ Algorithm." In 2018 3rd International Conference on Computer Science and Engineering (UBMK), pp. 382-385. IEEE, 2018.

[15] Too, Edna Chebet, Li Yujian, Sam Njuki, and Liu Yingchun. "A comparative study of fine-tuning deep learning models for plant disease identification." Computers and Electronics in Agriculture 2018.

[16] Barbedo, Jayme Garcia Arnal. "Impact of dataset size and variety on the effectiveness of deep learning and transfer learning for plant disease classification." Computers and electronics in agriculture 153 : 46-53.2018.

[17] Szegedy, Christian, Wei Liu, Yangqing Jia, Pierre Sermanet, Scott Reed, Dragomir Anguelov, Dumitru Erhan, Vincent Vanhoucke, and Andrew Rabinovich. "Going deeper with convolutions." In Proceedings of the IEEE conference on computer vision and pattern recognition, pp. 1-9. 2015.

[18] Erhan, Dumitru, Christian Szegedy, Alexander Toshev, and Dragomir Anguelov. "Scalable object detection using deep neural networks." In Proceedings of the IEEE conference on computer vision and pattern recognition, pp. 2147-2154. 2014.

\section{AUTHORS PROFILE}

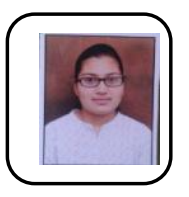

Satwinder Kaur is B.E in Electronics and Communication Engineering from Panjab University, Chandigarh, India. Here area of research includes image processing, plant disease detection. Currently, she is pursuing M.E from Panjab University, Chandigarh, India.

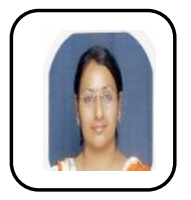

Garima Joshi is Ph.D. from Panjab University, Chandigarh, India. Her area of research includes image processing and VLSI design. She is working as Assistant Professor (ECE). She has more than 10 years of work experience. She has many publications in international Journals/Conference proceedings.

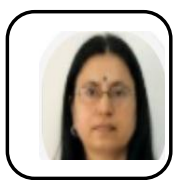

Renu Vig is Ph.D. from Panjab University, Chandigarh, India. Her area of research includes signal processing and soft computing techniques. Presently, she is working as Professor (ECE). She has more than 29 years of work experience. She has many publications in international Journals/Conference proceedings. 